\title{
Les microhabitats aquatiques des rives d'un grand cours d'eau : approche faunistique ${ }^{1}$
}

\author{
M. Bournaud ${ }^{2}$ \\ L. Cogerino ${ }^{2}$
}

Mots clés : Rive, grands cours d'eau, microrépartition, macroinvertébrés.

L'échantillonnage des rives aquatiques du Rhône, en amont de Lyon, fournit une approche faunistique nouvelle du milieu lotique. Les rives sont définies avec précision : la zone prospectée correspond à la frange supérieure de la rive immergée. Une mosaique complexe de microhabitats constitue la rive ; une liste non exhaustive en est proposé. Une description des techniques de prélèvement de chaque microhabitat est également fournie. Enfin une Analayse des Correspondances sur la matrice 35 relevés $\times \mathbf{8 0}$ taxons montre que les microhabitats abritent des communautés faunistiques effectivement différentes et caractéristiques des trois milieux d'érosion, de sédimentation et de végétation. La définition a priori des microhabitats est donc validée par la distribution des macroinvertébrés. Quelques exemples de taxons caractéristiques des différents microhabitats sont donnés: Trichoptères Leptoceridae en microhabitats d'èrosion, $H y d r o p s y c h e$ sp. dans les racines ou Oligochètes en microhabitat de sédimentation.

The aquatic microhabitats of the banks of a large water course : the faunistic approach.

Keywords : Banks, large rivers, microdistribution, macroinvertebrates.

The sampling of the submerged banks of the Rhône, upstream from Lyon, provided a faunistic approach that was new for the lotic environment. The banks a re precisely defined : the explored zone corresponding with the upper fringe of the submerged bank. A complex mosaic of microhabitats forms the bank and a non-exhaustive list is proposed. A description of the sampling techniques for each microhabitat is provided. Finally a correspondence analysis on a matrix of 35 surveys $\times 80$ taxa shows that the microhabitats shelter faunistic communities that are effectively different and characterised by three environments of erosion, sedimentation and vegetation. The a priori definition of the microhabitats is therefore validated by the distribution of the macroinvertebrates. Several examples are given of invertebrates that are characteristic of the different microhabitats : Leptoceridae (Trichoptera) in the eroding microhabitat, Hydropsyche sp. in the plant roots, and oligochaetes in the sedimenting microhabitat.

\section{Introduction}

La distribution des macroinvertébrés aquatiques est déterminée par un complexe de facteurs physicochimiques et biologiques (Hynes 1970, Friberg \& al 1977, Tolkamp 1982). Les facteurs géographiques (altitude, latitude, pente) déterminent les variables climatologiques et hydrauliques (en particulier la vitesse du courant, la température ou la composition chimique de l'eau) qui expliquent, au sein d'un même région, la répartition des animaux entre les différents cours d'eau (macrodistribution). Au sein d'une fraction de cours d'eau, substrat et quantité

1. Structure et fonct ionnement des écosystemes du Haut-Rhône français : 49 .

2. UA C.N.R.S. 367. Ecologie des eaux. Université Lyon I F 69622 Villeurbanne cedex. de nourriture disponible sont responsables de la microdistribution des macroinvertébrés aquatiques (Cummins \& Lauff 1969).

La partie aquatique des rives du Rhône, en amont de Lyon, présente une diversité de milieux bien supérieure à celle du chenal, constitué essentiellement de galets (Gaschignard \& El Hamdi 1984). De plus, ces milieux possèdent des caractéristiques originales, bien différentes de celles du chenal : faible profondeur, vitesses de courant plus faibles, dépôts de sédiments, substrats variés, souvent végétaux, etc... Il en résulte que ces rives, marges du milieu potamique lotique dominant, ont un rôle important pour le macrobenthos du chenal (Cellot 1982). Or les investigations antérieures ont porté principalement sur le chenal (Bournaud \& al. 1978, Cellot 1982, Gaschignard \& al. 1983. Bournaud \& al. 1986). Jusqu'à 
présent les rives ont été peu étudiées en tant que telles : les thavaux concernent essentiellement la faune interstitielle (Gilbert \& al. 1981, Dole 1983) ou la faune ripicole (faune macroscopique hygrophile et endogée d'origine terrestre) (Boumezzough 1983).

Une telle étude nécessite l'individualisation, sur le terrain, des micromilieux des rives immergées, définis a priori par leurs caractéristiques morphologiques. La valeur écologique de cette définition sera confirmée si ces habitats présentent effectivement des différences faunistiques.

\section{Définitions et méthode d'étude}

\subsection{Definitions des rives et de leurs microhabitats}

La rive joue le ròle d'écotone entre les milieux terrestres (terrestres s.str. et ripicoles) et aquatiques (interstitiels et potamiques). Par ailleurs la position de la rive aquatique d'un grand cours d'eau fluctue avec le niveau de l'eau. Nous définissons la partie aquatique de la rive de la manière suivante:

- Rive sensu lato: pour un niveau d'eau donné, la rive est la zone qui s'étend, en direction du chenal, depuis le bord même de l'eau jusqu'au secteur où le substrat change de nature (fig. 1). Lorsque la pente est faible, ce changement est souvent peu apparent. La rive sensu lato est alors définie comme la zone dont la limite inférieure est celle de la présence des hydrophytes (zone littorale de Dussart 1966). En l'absence de ceux-ci, par exemple avec une pente faible, un banc de galets et un courant rapide, la rive est très difficile à définir, du fait notamment de la grande superficie de fond découverte au moment des baisses de niveau. En pratique, on utilise alors la définition de la rive sensu stricto.

- Rive sensu stricto: Du point de vue faunistique, de nombreux auteurs (Mordukhai-Boltovskoi 1979, Cellot 1982, Biggs \& Malthus 1982) ont montré que biomasse, abondance et richesse faunist $i$. ques sont maximales dans la zone supérieure de la rive s.1. Nous limiterons donc nos investigations à cette zone supérieure, ou rive sensu stricto, d'ailleurs plus accessible et plus caractéristique de l'écotone étudié. Sa limite inférieure correspond à la projection sur le fond d'une distance horizontale de 1 mètre du bord de l'eau (fig. 1). Cette limite arbitraire
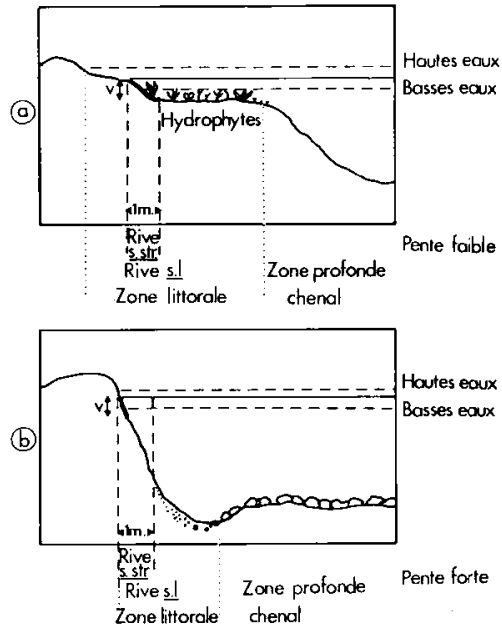

Fig. 1. Zonation transversale d'une rive
a) pente faible
b) pente abrupte
$v=$ zone prospecté, partie visible de la rive s.str.

se fonde sur l'observation que, hors de cette bande, la vitesse du courant notamment augmente très rapidement en direction du chenal. En pratique, notamment dans les cas d'une rive abrupte (fig. 1b), les investigations ne dépassent pas la limite inférieure de visibilité (variable selon la transparence de l'eau), soit environ 50 centimètres dans le cas du Rhône.

- Microhabitats : A la suite de Favet (1981) et Jenkins \& al. (1984), nous definissons trois types de micromilieux : les micromilieux d'érosion (= à substrat grossier et stable), les micromilieux de sédimen. tation (= à substrat fin) et les micromilieux de végétation.

Chacun regroupe plusieurs microhabitats définis par la granulométrie ou la végétation. La rive s.str. est constituée d'une mosaïque complexe de ces microhabitats. Le tableau 1 fournit la liste des 19 microhabitats rencontrés jusqu'ici sur les rives du Rhône. 


\subsection{Station d'étude : présentation}

Le canal de Miribel est un bras artificialisé du Rhóne, situé en amont de Lyon (Gaschignard \& al. 1983). Ce secteur de débit réservé de $30 \mathrm{~m}^{3} / \mathrm{s}$ est perturbé fréquemment et irrégulièrement, par des crues brutales dues aux manceuvres du barrage de Jons. La station retenue représente 500 mètres de rive droite abrupte où la profondeur d'eau varie de 0,30 à 1 mètre.

\subsection{Prélèvements faunistiques et variables relevées}

Les techniques classiques de prélèvement utilisées jusqu'ici en grand cours d'eau (Elliott \& Tullett 1978, 1983) (filet de Surber, cylindre de Hesse, substrats artificiels, dragues) ne peuvent être mises en ceuvre pour prospecter les microhabitats des rives. Les prélèvements sont donc effectués à l'aide d'instruments adaptés à chaque type de microhabitat : petites épuisettes triangulaires, grattoirs, cisailles, carottiers. La surface ou le volume inventorié est toujours le plus petit possible (dimension linéaire inférieure ou égale à 10 centimètres).

Le prélèvement a pour but d'extraire les macroinvertébrés du microhabitat prospecté. Cette opération s'effectue par râclage ou grattage du support in siru pour les microhabitats d'érosion, les excavations, les grosses branches ou racines $\left(n^{\circ} 13\right.$ et 14 , Tableau I). Le prélèvement lui-même est alors réduit aux organismes vivants sur le support et à quelques éléments fins le recouvrant (organiques ou minéraux). Lorsqu'il s'agit de supports sédimentaires $\left(\mathrm{n}^{\circ}\right.$ 4 à 8 , Tabl. I) ou végétaux ( $n^{\circ} 10$ à 12 et 15 à 19 , Tableau I) le microhabitat lui-même est prélevé avec les organismes qu'il abrite. La nature des éléments organiques ou minéraux prélevés est notée (variables $n^{\circ} 7$ et 8 . Tableau II). Les macroinvertébrés en sont extraits au laboratoire puis identifiés et dénombrés.

Trois prélèvements de faune sont effectués dans chacun des 12 types de microhabitats présents sur 500 mètres de rive de la station étudièe (25 février 1985). Chacun des 35 relevés faunistiques effectivement réalisés est accompagné de l'enregistrement de 8 variables qualitatives (Tableau II). Outre le type de microhabitat (variable qualitative $n^{\circ} 1$ ), cinq de ces variables définissent, en classes qualitatives, le milieu au niveau du prélèvement (profondeur, vitesse du courant, présence de périphyton, écart de température, conductivité). Deux autres variables $\left(n^{\circ} 7\right.$ et 8 ) précisent la nature du substrat effectivement prélevé, principal et secondaire.

\section{Résultats}

\subsection{Interprétation}

Les 35 relevés effectués fournissent une liste faunistique de 80 taxons. Une analyse des Correspondances sur la matrice 35 relevés $\times 80$ taxons met en évidence les ressemblances faunistiques des rele. vés. Des points relevés sont d'autant plus proches sur les axes qu'ils contiennent des peuplements plus semblables. Ainsi le prélevement des 35 points relevés sur le plan factoriel F1 F2 de leurs coordonnées (fig. 2) permet de rapprocher les points ayant les plus grandes ressemblances faunistiques. La projection sur ces points des variables les concernant, en particulier le type du microhabitat (variable $n^{\circ} 1$, Tableau II), montre que les relevés provenant de microhabitats semblables ou de nature voisine sont faunistiquement proches.

Cette image fait en effet apparaître clairement trois pôles de ressemblances : les microhabitats de sédimentation (surtout sables, limons, graviers), les microhabitats d'érosion (pierre et blocs) et les microhabitats végétaux (sutout les branches). Les racines et les excavations se rapprochent du centre : leur faune est moins particulière. Cette typologie faunistique des prélèvements montre que les microhabitats tels que nous les avons définis a priori présentent des caractéristiques propres auxquelles la faune est sensible. Le regroupement en trois types de micromilieux (Tableau I) est ainsi également validé du point de vue faunistique (fig. 3a).

De façon identique, la projection des sept autres variables de milieu (Tableau II) sur ce mème plan fournit les caractéristiques des groupes de relevés. Ces relations n'apparaissent nettement que pour deux des variables enregistrées, la nature du substrat principal prélevé dans le microhabitat (variable $n^{\circ} 7$, Tableau II) et la présence de périphyton (variable $n^{\circ} 6$ ) ( $f$ ig. 3 b et c). On remarque ainsi que le substrat principal prélevé, examiné au moment du tri des organismes, dépend de la nature du microhabitat. En effet, si on prélève toujours des éléments végétaux (débris, branches) dans les microhabitats 
Tableau I. Définition des microhabitats des rives immergées du Rhône et leurs abréviations.

Microhabitots retenus

\begin{tabular}{|c|c|c|c|c|}
\hline & Nam & Diamètre des éléments & Code & Micramilieu \\
\hline 1 & Dalles &, $100 \mathrm{~cm}$ & Oa & \\
\hline 2 & Blocs & $25-100 \mathrm{~cm}$ & 81 & Erosion ER \\
\hline 3 & Pierres & $3,2-25 \mathrm{~cm}$ & $P$ & \\
\hline 4 & Groviers & $0,2-3,2 \mathrm{~cm}$ & 0 & \\
\hline 5 & Sables & $600 \mu-2 \mathrm{~mm}$ & $S$ & \\
\hline 6 & Limon/pierre & $40-600 \mu$ & $L$ & Sédimentation SE \\
\hline 7 & Limon-sable & $40-600 \mu$ & 1 & \\
\hline 8 & Argiles & $(40 \mu$ & Ar & \\
\hline \multicolumn{5}{|c|}{--------1} \\
\hline 9 & Excavation (covité nue, & & & \\
\hline & sous blocs, racines...) & & $\mathbf{E}$ & \\
\hline 10 & Débr is (feuilles, tiges...) & & Dé & \\
\hline 11 & Racines & $0<5 \mathrm{~cm}$ & $r$ & \\
\hline 12 & Branches & $0<5 \mathrm{~cm}$ & b & \\
\hline 13 & Racines & $9>5 \mathrm{~cm}$ & $\mathbf{R}$ & \\
\hline 14 & Branches & $9>5 \mathrm{~cm}$ & B & Végétation YG \\
\hline 15 & Chevelu (racinaire) & & C & \\
\hline 16 & Hélophytes & & Hé & \\
\hline 17 & Hydrophytes & & Hy & \\
\hline 18 & Bryophytes & & $\mathrm{Br}$ & \\
\hline 19 & Algues filamenteuses & & $\mathbf{A l}$ & \\
\hline
\end{tabular}


Tableau II. Variables qualitatives enregistrées au niveau d'un prélèvement faunistique. Les modalités utilisées pour la profondeur, la vitesse du courant et la température sont des classes qualitatives d'évaluation rapide de ces variables quantitatives.

\begin{tabular}{|c|c|c|c|c|c|c|c|c|c|}
\hline & & \multicolumn{8}{|c|}{ VARIABLES } \\
\hline & & $\begin{array}{c}\text { Nicrohabitat } \\
\text { prospecte }\end{array}$ & Profondeur & $\begin{array}{l}\text { Vitesse du } \\
\text { courant } \\
\text { (1) }\end{array}$ & $\begin{array}{c}\text { Temperature } \\
(2)\end{array}$ & $\underset{\mu \mathrm{H} / \mathrm{km}}{\text { Conductive }}$ & Périohyton & $\begin{array}{l}\text { Substrat } \\
\text { principal } \\
\text { preleve }\end{array}$ & $\begin{array}{c}\text { Substrat } \\
\text { seconda ire } \\
\text { prélevé }\end{array}$ \\
\hline 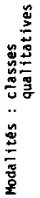 & $\begin{array}{r}1 \\
2 \\
3 \\
4 \\
5 \\
6 \\
7 \\
8 \\
9 \\
10 \\
11 \\
12\end{array}$ & $\begin{array}{l}\text { BToc } \\
\text { Pierre } \\
\text { gravier } \\
\text { sable } \\
\text { Limon-pierre } \\
\text { Limon-sable } \\
\text { Excavation } \\
\text { Racines \& } 5 \mathrm{~cm} \\
\text { Branches \& } 5 \mathrm{~cm} \\
\text { Branches } 5 \mathrm{~cm} \\
\text { Chevelu } \\
\text { Algue }\end{array}$ & $\begin{array}{r}0=15 \mathrm{~cm} \\
.15 \mathrm{~cm}\end{array}$ & $\begin{array}{l}\text { nulle } \\
\text { tres lente } \\
\text { lente } \\
\text { rapide }\end{array}$ & $\begin{array}{r}0=0,6 \\
0,6=1,2 \\
1,2=4,8\end{array}$ & $\begin{array}{l}230=430 \\
430=530\end{array}$ & $\begin{array}{l}\text { absent } \\
\text { present }\end{array}$ & $\begin{array}{l}\text {-pierre } \\
\text { - sable } \\
\text { - limon-sable } \\
\text { - debris } \\
\text { - semblable } \\
\text { au micro- } \\
\text { habitat }\end{array}$ & $\begin{array}{l}\text {.oierre } \\
\text { gravier } \\
\text { e.sable } \\
\text {.limon-sable } \\
\text {.argile } \\
\text {.debris }\end{array}$ \\
\hline
\end{tabular}

(1) nulle = le 1 imon agite reste sur place

très lentẹ = le limon agité se déplace a peine

lente = lé limon agité se déplace avec une vitesse estimée de 1 'ordre de $10 \mathrm{~cm} / \mathrm{s}$

rapide = le limon agité se déplace avec une vitesse estimée superíeure a $10 \mathrm{cn} / \mathrm{s}$

(2) écart à la température ninimale

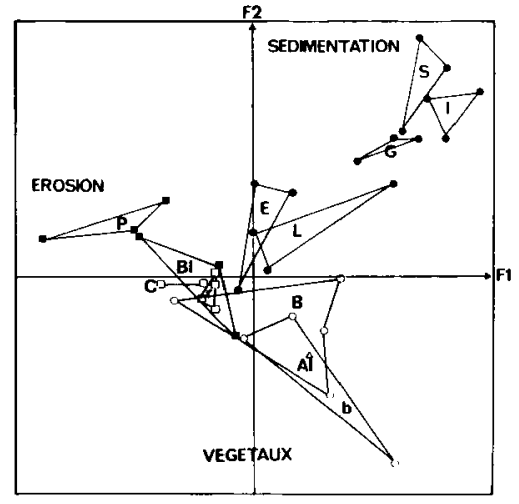

Fig. 2. Structure des relevés dans le plan faunistique F1F2 d'une Analyse des Correspondances (35 relevés $\times 80$ taxons). Projection des modalités de la variable * nature du microhabitat "sur chacun des 35 relevés. (Abréviations cf. Tableau I).

Microhabitats - carré noir : d'érosion - rond noir : de sédimentation.

Végétaux - carré blanc : racines - rond blanc : branches - triangle blanc : algues. végétaux, on prélève très souvent du limon (sédiment) dans les microhabitats minéraux, d'érosion et de sédimentation (moitié supérieure de la figure). Toutefois on prélève des pierres uniquement dans les microhabitats de sédimentation. Il en résulte une complexité granulométrique plus grande dans les prélè. vements sur microhabitats minéraux par rapport aux prélèvements sur microhabitats de branches. Enfin, le périphyton n'est normalement présent que sur les substrats les plus stables, soient les branches ou les substrats d'érosion (pierres, blocs) (fig. 3c).

La répartition des taxons dans le plan dont on vient d'interpréter la signification mésologique concrétise la relation faune-microhabitats (fig. 4). La figure 41 donne neuf exemples types de distribution faunistique par rapport aux microhabitats, véritable profil d'habitat des éléments faunistiques correspondants, dont les plus caractéristiques sont énumérés sur la figure 5.

\subsection{Rôle du substrat dans la microdistribution des macroinvertébrés des rives}

Le problème des relations organismes-substrat, en milieu lotique, a déjà fait l'objet de nombreux

1. La figure 4 a été exécutée à l'aide du logiciel Graphique de Auda (1983). 


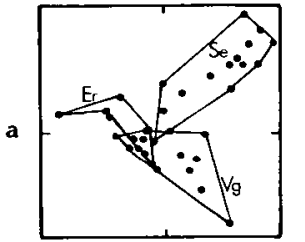

mineral

Microhabitat

vegetal

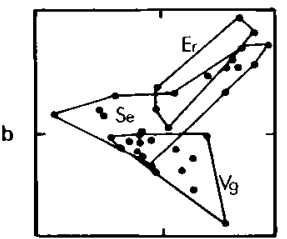

Substrat principal prélevé

\section{Periphyton}

c

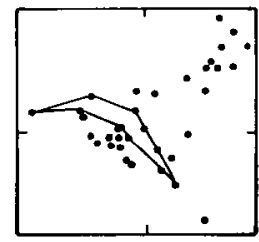

Fig. 3. Projection de variables caractérisant les 35 relevés sur le plan faunistique défini sur la figure 2.

a) Micromilieu.

b) Nature du substrat principal prélevé

c) Présence de périphyton

Se $=$ sédinent

$E r=$ milieu d'érosion

$\mathrm{Vg}=$ végétaux

articles généraux (Cummins \& Lauff 1969, Hynes 1970, Prodon 1976). Parmi les variables mesurées ici, c'est le type de substrat qui apparaît comme l'élément essentiel de la définition du microhabitat. C'est lui qui, en effet, explique le mieux la distribution des macroinvertébrés.

- Les milieux d'érosion (blocs et pierres) présentent une grande complexité spatiale, des abris en abondance (interstices de grande taille). Ce sont les seuls substrats minéraux présentant une couverture de périphyton. La richesse faunistique y est élevée (fig. 6). Les Trichoptères Leptoceridae y sont particulièrement représentés notamment par leurs jeunes stades (Oecetis, Triaenodes). La faible rhéophilie de ce groupe détermine en partie son abondance dans ces microhabitats de rives, toujours abrités, en relation avec la ponte ou la nymphose (Kuusela 1979).

- Les milieux de sédimentation (gravier, sable, limon): les interstices sont accessibles uniquement à la faune de faible dimension, aux jeunes stades larvaires d'insectes, et aux Oligochètes (Mackay 1969, Khalaf 1975). Le limon et le sable par leur absence d'abris, sont donc défavorables à de nombreux éléments de la macrofaune (Maitland 1964). Le dépôt de limon sur les pierres (Prodon 1976) colmate les interstices et fait disparaître le périphyton. La richesse faunistique est donc faible dans les microhabitats de sédimentation (fig. 6).

- Les milieux de végétation apparaissent comme les plus riches (fig. 6). Ils constituent des supports sur lesquels se déposent des éléments surtout organiques. Les racines constituent, à cet égard, un véritable filtre le long des rives où s'accumulent les débris organiques, jamais cependant en grande quantité. Si leur structure spatiale complexe offre un grand nombre d'abris, les racines sont toutefois relativement bien exposées au courant, qui assure un bon renouvellement de l'oxygène. Elles renferment essentiellement cinq espèces de Trichoptères Hydropsychidae (fig. 5), des Gammares. Ces taxons relativement rhéophiles recherchent ici les abris et leur stabilité, tout en profitant des apports nutritifs dus aux courants (larves filtreuses d'Hydropsyche).

Sur les branches, les surfaces exposées au courant sont plus importantes. La faune y est presque exclu. sivement rhéophile. Les trois espèces de Baetis récoltées préfèrent ces substrats végétaux aux pierres trop recouvertes de sable et limon. D'autres, comme les larves du Coléoptère Orectochilus villosus et des Diptères Stenochironomus trouvent leurs abris ou leur nourriture dans le bois. La larve d'Hydropsyche siltalai est la seule de la famille des Hydropsychidae dont le préfèrendum est situé dans les branches : c'est aussi la plus rhéophile de cette famille.

Le cas du Coléoptère Esolus parallelepipedus est remarquable. Les larves sont présentes dans une grande variété de microhabitats (fig. 4) avec une préférence pour les milieux minéraux, tandis que les imagos ont un habitat restreint aux végétaux et 
Axe 2

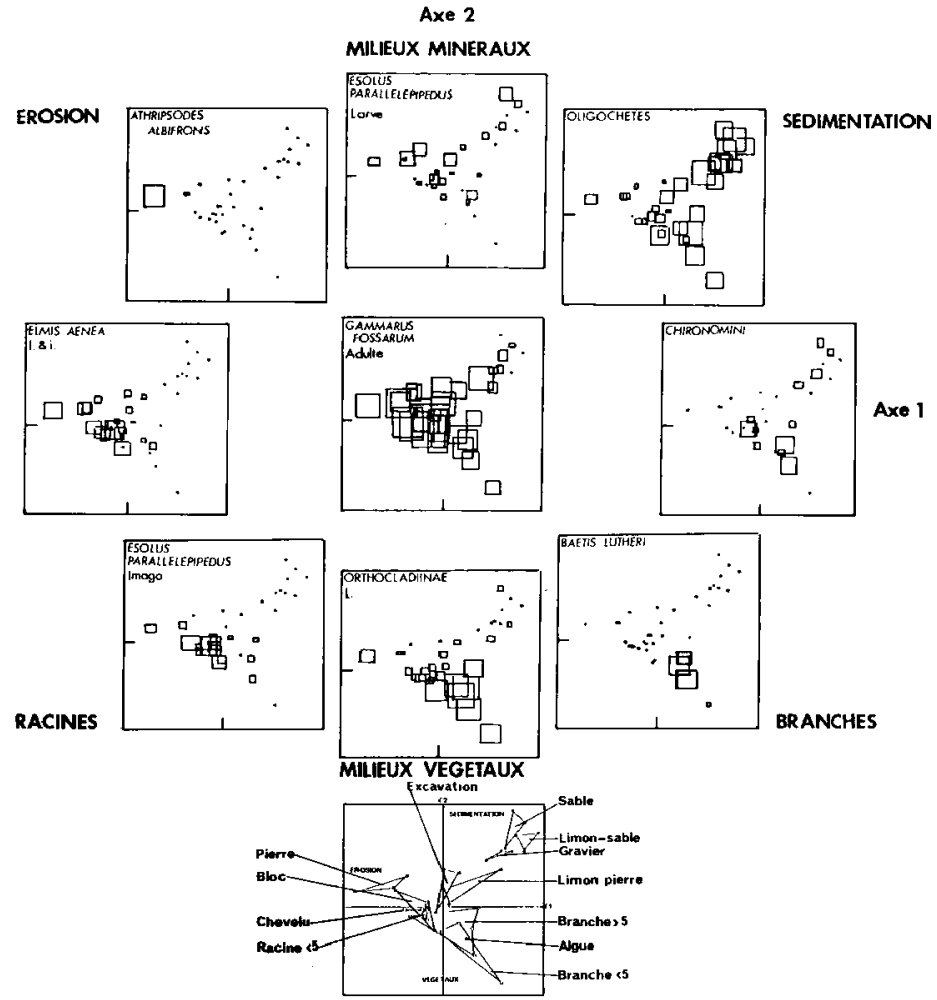

Fig. 4. Quelques exemples de répartition des taxons dans les microhabitats et micromilieux des rives. Les 35 relevés sont disposés dans le plan F1F2 interprété sur les figures 2 (rappelée ici en bas) et 3. Les dimensions des carrés sont proportionnelles à l'abondance des taxons dans chaque relevé. L'absence d'un taxon est figurée par un point (1) = larve, (i) = imago.

surtout aux racines: il y a diminution de l'amplitude d'habitat après passage à l'état adulte.

\section{Conclusion}

Les microhabitats les plus pauvres en espèces (fig. 6) sont ceux de sédimentation. C'est là que le colmatage des interstices des pierres par le limon ou la compacité du limon-sable sont les plus forts et que la vitesse du courant est la plus faible.

Les différences essentielles de peuplement observées entre les microhabitats peuvent s'interpréter en grande partie par des différences de stabilité, par exemple entre pierres et végétaux ou racines et branches (fig. 5). C'est d'ailleurs aussi une différence 


\section{EROSION \\ T Althripsates allitirons (1) \\ T ceralea dissimilis (1) \\ I Polytentrupus novomazlatus (1) \\ T azetis larvules \\ T Trianates larvules \\ M Ancylus fluviatilis \\ C Elmis aeneo (1 et i) \\ C Riolus subviolaceus ( 1 )}

$\operatorname{axe} 2 \uparrow$

(milieuX MINERAUX)

c Esolus porallelepipodus (1)

\begin{tabular}{|c|c|c|c|c|}
\hline & $\mathbf{T}$ & Alhripsodes lervules & & axe 1 \\
\hline & $\mathbf{E}$ & Coenis luctuosa ( 1 ) & & \\
\hline I Cheumatopsyche lepids (1) & & & & Boetis lutheri (1) \\
\hline T Mporopsyche modesio (1) & & & & Balis rhadani (1) \\
\hline I Hadropsyche pellucidula( (1) & & & C & Macronychus quadr ituberculotus(1) \\
\hline T Mudropsyche contubernslis (1) & & & & Orectochilus villosus (1) \\
\hline I Hydropssthe exocellata (1) & & & & Simuliidae (1) \\
\hline T Halesus radialus (1) & D & Orthoclodinge (1) & & Tanytorsini $(\mathrm{n})$ \\
\hline c Oulimnius tuberculatus ( 1 et i) & D & ronytorsini (1) & & Stenactironomus (1) \\
\hline c Esolus porallelepipedus (i) & D & Tonypodinoe (1) & & Orthocladinge $(\mathrm{n})$ \\
\hline \multirow[t]{2}{*}{$\mathrm{Cr}$ Commoruspulex( $\mathrm{i})$} & D & \multicolumn{3}{|l|}{ Antacha vitripennis (1) } \\
\hline & $\mathrm{T}$ & \multicolumn{3}{|l|}{ Psychomyig pusilis (1) } \\
\hline \multirow[t]{3}{*}{ RACINES } & $\mathbf{I}$ & \multicolumn{3}{|l|}{ Hodropsyche silloloi (1) } \\
\hline & $\mathbf{E}$ & \multirow{2}{*}{\multicolumn{3}{|c|}{$\begin{array}{l}\text { Heptogenia sulphures ( } 1 \text { ) } \\
\text { (milieux VEGE IAUX) }\end{array}$}} \\
\hline & & & & \\
\hline
\end{tabular}

Fig. 5. Taxons correspondants aux profils écologiques des microhabitats de la figure 4.

Les taxons soulignés sont ceux représentés sur la figure 4 .

Les taxons dont l'abondance est inférieure à 4 individus, ou dont la distribution n'est pas nette, sont éliminés (seuls 39 taxons sont conservés). (l.) : larve ; (i) : imago.

C : Coléoptères ; T : Trichoptères : M : Mollusques ; Cr: Crustacés ; D : Diptères ; E : Ephéméroptères 

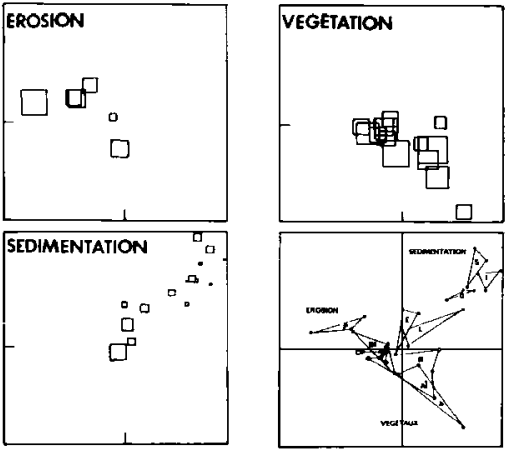

Fig. 6. Richesse faunistique des microhabitats répartis en micromilieux d'érosion, de végétation et de sédimentation. La taille des carrés est proportionnelle à la richesse. La figure 2 est rappelée en bas à droite.

de stabilité qui explique l'essentiel des différences faunistiques entre rives et chenal.

En définitive le découpage en microhabitats, tels que nous les avons définis initialement, s'avère pertinent du point de vue faunistique, puisque les macroinvertébrés se répartissent en fonction du type même de ces habitats. Les autres variables mesurees (Tableau II) apparaissent en effet beaucoup moins synthétiques : leur pouvoir explicatif de la microrépartition faunistique est beaucoup plus faible (fig. 3). Ainsi reconnaître extérieurement le type de microhabitat équivaut à reconnaitre le type de faune qu'il contient.

La perspective d'une étude des rives de grand cours d'eau à travers les microhabitats ainsi définis s'avère alors être une voie d'approche commode par la relative simplicité technique face à un ensemble très hétérogène et informative par la richesse de ces microhabitats en descripteurs faunistiques de fonctionnement (Bournaud \& Amoros 1984). Il conviendra donc d'utiliser cette voie pour comprendre les relations fonctionnelles rives-chenal-sousécoulement dans l'espace eupotamique.
Travaux cités

Auda (Y.). 1983. - Róle des méthodes graphiques en analyse des données: application au dépouillement des enquêtes écologiques. Thèse $3 e$ cycle. Lyon I : $127 \mathrm{p}$.

Biggs (B.J.) \& Malthus (T.J.). 1982. - Macroinvertebrates associated with various aquatic macrophytes in the backwaters and lakes of Upper Clutha Valley, New Zealand. N. Z. J. Mar. Freshw. Res. $16: 81-88$.

Boumezzough (A.). 1983. - Les communautés animales ripicoles épigées et endogées du bassin versant de la rivière Aille (Var.

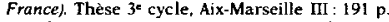

Bournaud (M.) \& Amoros (C.). 1984. - Des indicateurs biologiques aux descripteurs de fonctionnement : quelques exemples dans un système fluvial. Bull. Ecol., 15 (1): 57-66

Bournaud (M.), Chavanon (G.) \& Tachet (H.). 1978. - Structure et fonctionnement des écosystèmes du Haut-Rhône français. 5. Colonisation par les macroinvertébrés de substrats artificiels suspendus en pleine eau ou posés sur le fond. Verh. Intemat. Ver, Intemat. Ver. Limnol, 20: 1485-1493.

Bournaud (M.), Tachet (H.), Roux (A.L.) \& Auda (Y.). 1986. - The effects of seasonal and hydrological influences on the macroin. vertebrates of the Rhone river. France. En preparation.

Cellot (B.). 1982. - Cycle annuel at zonation de la derrive des macroinvertébrés du Rhóne en amont de Lyon. These 3 cycle, Lyon I : $167 \mathrm{p}$.

Cummins (K.W.) \& Lauff (G.H.). 1969. -- The influence of substrate particle size on the microdistribution of stream macrobenthos. Hydrobiologia. 34 (2) : $145-181$.

Dole (M.J.). 1983. - Le domaine aquatique souterrain de la plaine alluviale du Rhóne à l'est de Lyon 1. Diversité hydrologique et biocénotique de trois stations représentatives de la dynamique fluviale. Vie Milieu, 33 (3/4) : 219.229.

Dussart (B.). 1966. - Limnologie, l'étude des equx continentales. Paris, Gauthier-Villars : $676 \mathrm{p}$.

Elliott (J.M.) \& Tullett (P.A.). 1978. - A bibliography of samplers for benthic invertebrates. Occ. Publs. Freshwat. Biol. Ass., 4, $61 \mathrm{p}$.

Elliot (J.M.) \& Tullett (P.A.). 1983. - A supplement to a bibliography of samplers for benthic invertebrates. Occ. Publs. Freshwat. Biol. Ass., 20, $27 \mathrm{p}$

Favet (C.) 1981 . - Communautés nipicoles de la basse Durance, These de 3e cycle, Aix-Marseille 3:371 p

Friberg (F.), Nilsson (L.M.), Otto (C.), Sjöströrn (P.), Svensson (B.W.) \& Svensson (B.J.). 1977. - Diversity and environments of benthic invertebrate communitics in south Swedish streams. Arch. Hydrobiot., 81 (2) : $129-154$

Gaschignard (O.) \& El Handi (M.). 1984. - La granulométrie du sédiment ef l'évolution du benthos dans un bras vif du Rhóne. Rev. fr. Sc, eau, $3: 279-293$.

Gaschignard (O.), Persat (H.) \& Chessel (D.). 1983. - Répartition transversale des macroinvertébrés benthiques dans un bras du Rhône. Hydrobiologia. 106: 209-215.

Gibert (J.), Ginet (R.). Mathieu (J.) \& Reygrobellet (J.L.). 1981. Structure et fonctionnement des écosystèmes du Haut-Rhòne français : 9 - Analyse des peuplements de deux stations phréa. tiques alimentant des bras morts. Int. J. Speleol., 11 : 141-158.

Hynes (H.B.N.), 1970. - The ecology of nonning waters. Liverpool University Press : 555 p.

Jenkins (R.A.), Wade (K.R.) \& Pugh (E.). 1984. Macroinvertebrate-habital relationship in the River Teifi catchment and the significance to conservation. Freshwat. Biol, 14 (1) : $23-42$.

Khalaf (G.). 1975. - Utilisation des substrats antificiels en eau courante pour l'étude de la répartition et de la dynamique de colo nisation des macroinvertébrés benthiques. Thèse 35 cycle, Lyon I : 91 p. 
Kuusela (K.). 1979. - Early summer ecology and community structure of the macrozobenthos on stones in the Jäväjänkoski rapids on the river Lestijoki. Finland. Acta Univ. Oul., A 87 (6) : $123 \mathrm{p}$.

Mackay (R.J.). 1969. - Aquatic insect communities of a small stream on Mt Saint-Hilaire (Quebec). J. Fish. Res. Board. Can., 26 (5): $1157 \cdot 1183$.

Maitland (P.S.). 1964. - Quantitative studies on the invertebrate fauna of sandy and stony substrates in the River Endrick, Scotland. Proc. r. Soc. Edimb., (B-4) : 277-301.
Mordukhai-Boltovskoi (Ph. D.). 1979. - The river Volga and its life: Zoobenhos and other invertebrates living on substrata. The Hague, Dr W. Junk : 475 p.

Prodon (R.). 1976. -- Le substrar, facteur écologigue el éthologique de la vie aquatique : observations ex expériences sur les larves de Microptema testacea et Cordulegaster annulatus. Thèse $3^{\text {c }}$ cycle, Lyon I : 221 p.

Tolkamp (H.M.). 1982. - Microdistribution of macroinvertebrates in lowland streams. Hydrobiol. Bull., $16(2.3): 133-148$. 